\title{
Mechanisms meet Structural Explanation
}

\author{
Laura Felline*
}

\begin{abstract}
This paper investigates the relationship between Structural Explanation and the New Mechanistic account of explanation. The aim of this paper is twofold: firstly, to argue that some phenomena in the domain of fundamental physics, although mechanically brute, are structurally explained; and secondly, by elaborating on the contrast between SE and ME, to better clarify some features of SE. Finally, this paper will argue that, notwithstanding their apparently antithetical character, SE and ME can be reconciled within a unified account of general scientific explanation.
\end{abstract}

Keywords. Structural Explanation, Mechanistic Explanation, Epistemic Conception of Explanation.

\section{Introduction}

One of the major strengths of the New Mechanistic philosophy (Glennan 1996, Machamer et al. 2000 ) is the virtually ubiquitous application of mechanistic reasoning in scientific practice, which also makes Mechanistic Explanation (ME) plausibly the most successful account of scientific explanation currently available. However, there are some domains where mechanistic reasoning, and ME with it, drastically loses its predominance in the scientific enterprise. This is often the case in the philosophy of fundamental physics. On the other hand, those phenomena which are not explained through a ME are not necessarily unexplainable brute facts of nature: mechanically brute (as I will call them) phenomena might be nonetheless explained by science. One partial aim of this paper is to give flesh to this claim by showing that some mechanically brute phenomena in the domain of fundamental physics are structurally explained.

Since its origin in R.I.G. Hughes' work (Hughes 1989b, p.175), a central role in the definition of Structural Explanation (SE) was played by the claim that SE is independent of any assumption about what types of entities and what types of processes lie within the theory's domain. On the one hand, this feature received an essentially anti-metaphysical connotation; on the other, it was also

\footnotetext{
* Department of Philosophy, Università Roma Tre, via Ostiense 234, 00146 Rome, Italy. E-mail: fellinelaura [at]
} gmail . com 
used as the key to understand the non-causal character of SE. Some central features of SE have therefore been shaped on the elaboration of such a characterization. The second partial aim of this paper is to reconsider Hughes' original characterization of the non-causal character of SE as a contrast with a merely metaphysical kind of causal explanation, and to re-qualify it as a contrast with ME.

The paper's structure is as follows. In $\S 1$, I introduce the issue of the limited applicability of ME in fundamental physical theories. Probably the mechanistic philosopher who has devoted the most attention to such problem is Stuart Glennan (1996, 2002, 2010, and more recently Kuhlman and Glennan 2014), so his framing of the issue deserves a special attention. Independent of more general considerations, in $\S 2$ I provide two illustrating examples of phenomena that are typically seen as successfully explained by our best fundamental physical theories and yet, I will argue, are mechanically brute. More specifically, such phenomena are structurally explained. In $\S 3$, I elaborate on the contraposition between SE and ME and investigate the consequences of such contraposition for SE. It could be argued that the two accounts of explanation refer to antithetical general views of scientific explanation and cannot therefore be both true. To counter such a conclusion, in $\S 4$ I underline the common features of SE and ME and show that they might be assimilated by the same theory of explanation. More exactly, ME and SE can be both assimilated by an epistemic, modelbased account of explanation that work by providing information about patterns of counterfactual dependences.

As I have said, the aim of this paper is to investigate the relationship between SE and ME. I will not in general be concerned by the question as to whether SE is also mutually exclusive to other kinds of causal explanation (of causal relevance or of other kinds of causal production), nor whether fundamental physics provides causal explanations different from ME of some mechanistically brute phenomena. There is a recent growing literature about non-causal and so-called mathematical explanations in natural sciences (e.g. Batterman and Rice (2014), Reutlinger (2012), Pincock (2013), Lange (2013), Skow (2013)). The issues at the centre of this debate are very important and intersect in some points with the literature on SE. However, in this paper I will only occasionally touch on such arguments. I have left until another occasion the study of how SE inserts in these important topics. 


\section{Mechanisms and the explanation of fundamental phenomena}

Mechanically explaining a phenomenon P requires describing, in a more or less idealized way, the mechanisms responsible for P (Glennan 2002, Bechtel and Abrahamsen 2005, Craver 2007, Illari and Williamson 2010). The novelty of the New Mechanistic philosophy, with respect to the traditional mechanical philosophy of science, lies in its definition of mechanisms as systems. There is actually no consensus yet on the correct definition of mechanism, and the details of this debate are irrelevant to the scope of this paper. Here it is sufficient to cite two of the most quoted:

A mechanism for a behavior is a complex system that produces that behavior by the interaction of a number of parts, where the interactions between parts can be characterized by direct, invariant, change-relating generalizations. (Glennan 2000, p. S344)

And:

Mechanisms are entities and activities organized such that they are productive of regular changes from start or set-up to finish or termination conditions. (Machamer et al. 2000, p. 3)

A common feature of all different characterizations of mechanisms as systems is that they are hierarchical, in the sense that each part of a mechanism is a mechanism in itself and its behavior is therefore explainable by the description of its components and the interaction between them (Glennan 2011, Machamer et al. 2000).

This process of regress, though, is not infinite. At one point, one reaches a level where mechanistic reasoning and $\mathrm{ME}$ have no place. This follows, under an atomist stance, straightforwardly by assumption. If the layers of physical composition have a bottom level, i.e., the level of the most elementary components of the world - then, by assumption, the behaviour of such elements cannot be explained in terms of the interaction between their component parts (Glennan 1996, 2002, 2010). Anyway, regardless of whether or not one adopts an atomist stance, mechanistic reasoning - and ME with it - drastically loses its ubiquitous role at the level of current fundamental physics. The question of exactly where ME is or not applicable obviously depends on the specific details of the mechanistic account chosen. For instance, (Kuhlman and Glennan 2014) argued that mechanistic reasoning is generally not applicable to quantum phenomena because the concept of entities as parts of mechanisms might be problematic in (at least) three ways. According to them, in fact, at least three characteristic features of quantum systems (i.e. indeterminateness of properties, indeterminateness of position, entanglement) are incompatible with mechanistic reasoning.

First of all, while in ME the behaviour of a mechanism depends on the interaction of its parts in virtue of their dynamical properties, the vast majority of properties of a quantum object are virtually always indeterminate. MEs that depend on such dynamical properties are therefore in general forbidden in quantum phenomena. 


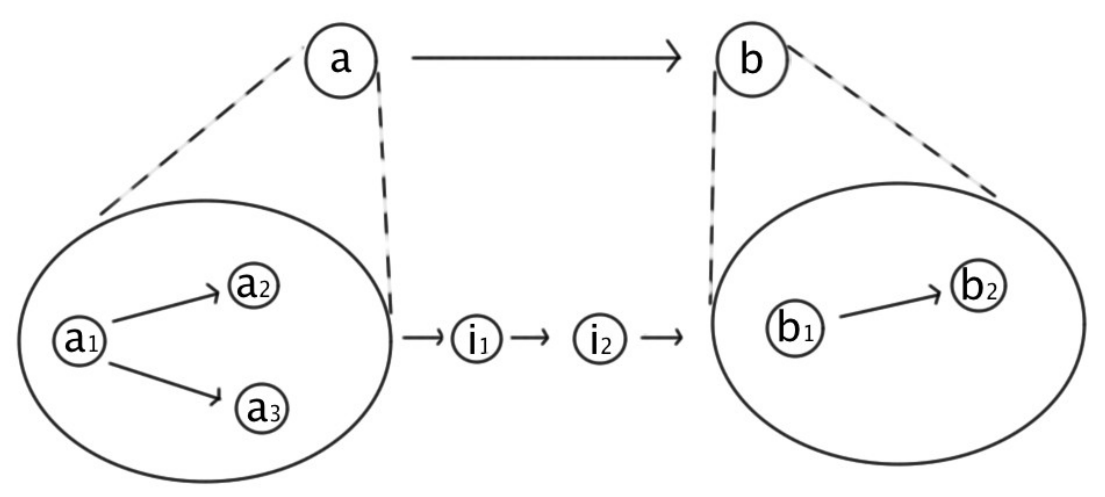

Figure 1. (Redrawn from Glennan (2011)) The two parts $a$ and $b$ of $a$ mechanism are mechanisms themselves. The arrows represent the interaction between parts $a$ and $b$ of a mechanism, and the dashed lines represent constitutive relations. $a$ and $b$ are themselves complex systems, mechanisms whose behavior is explainable by the behavior and interactions of their constituents (as the way they interact, which can also involve the mediation of further entities ( $i_{1}$ $\left.\mathrm{i}_{2}\right)$ ).

Secondly, among such properties, one of the most important in ME, due to its role in the organization between a mechanism's component parts, is the spatial location of such parts. In general, though, quantum objects are not describable as localized objects. As a consequence, this feature of ME is also generally problematic. Third, quantum objects are virtually always in entangled states. When a composed system is in an entangled state, the latter state is not captured by the states of the component systems plus their spatio-temporal organization. Moreover, in an entangled state, each individual component system does not have a determinate quantum state, only the composed system does. The behavior of a system composed of two entangled subsystems is therefore mechanically unexplainable for two reasons: on the one hand, in a ME a complex system must be decomposable in the sum of its parts; on the other, a ME requires the possibility of individuating parts with individual states.

Obviously, it could be argued that ME does not necessarily require determinateness of properties, especially of location, and factorizability - in the next section I will illustrate with two case studies that, independent of the specific features one attaches to ME, there are some explanations of fundamental physical phenomena that are not ME.

In Glennan's older works the conclusion of the analysis of the case of mechanical inexplicability 
of the fundamental laws of physics was that such laws are "brute nomological facts of our universe" (2002, S348, but see al1996). ${ }^{1}$ However, phenomena that are not mechanistically brute are not necessarily unexplainable brute facts of our universe, unless one assumes that the only genuine scientific explanation is ME. In the pluralistic view defended in this paper, a phenomenon can be mechanically brute but still explained by science. There are indeed many cases of mechanically fundamental phenomena that are typically conceived as explained by science. ${ }^{2}$ In particular, as I am going to argue in the next section, some of them are structurally explained.

\section{Two examples of SE}

The structural account of explanation was originally outlined by R.I.G Hughes (1989a, b) and successively developed by Clifton (2001), Bokulich (2009), Felline (2010) and Dorato and Felline (2011). At a later point ( $\$ 4)$, I will articulate more precisely some of its features. For the moment it is sufficient to know that SE is a model-based explanation (Hughes (1997)) that exploits the mathematical models displayed by the theory and works by showing how (the formal representative of) the explanandum counterfactually depends on other mathematical elements or features of the model (Bokulich (2009)).

The geometrical explanations of relativistic effects in the Special Theory of Relativity provide clear-cut and well-known examples of phenomena that are structurally explained. To illustrate in more detail how such explanations work, let us take the example of length contraction. This is the phenomenon for which the length of a rod, when measured by an observer in relative inertial motion, is shorter than its proper length.

The most convenient representation of phenomena in Special Relativity makes use of Minkowski diagrams. So, first of all, the fundamental quantity in Minkowski space-time is the (pseudo) distance between two events, namely the space-time interval $l$. For simplicity, let's put one of such events at the origin $\mathrm{O}$ and the other in the event $\mathrm{A}(\mathrm{x}, \mathrm{t})$ of the reference frame $\mathrm{S}$. Then the space-time interval OA is given by the formula

$$
\mathrm{I}^{2}=\mathrm{x}^{2}-\mathrm{t}^{2}
$$

This formula for the 'distance' between two points represents the fundamental difference between Minkowski space-time and Galilean space-time and is originated by the different geometries they adopt. While in the Galilean (Euclidian) space-time the distance between two points (events) OA follows the Pythagorean theorem:

$$
\text { distance }^{2}=\mathrm{x}^{2}+\mathrm{y}^{2}
$$

in Minkowskian space-time, such a theorem is not valid and is replaced by its corresponding

1 Kuhlmann and Glennan (2014) though have recently adopted a more explicitly pluralistic position.

2 Of course I am not denying here the existence of basic unexplained facts at the bottom of our scientific explanations.

That some fundamental phenomena are structurally explained does not mean that all of them are. 


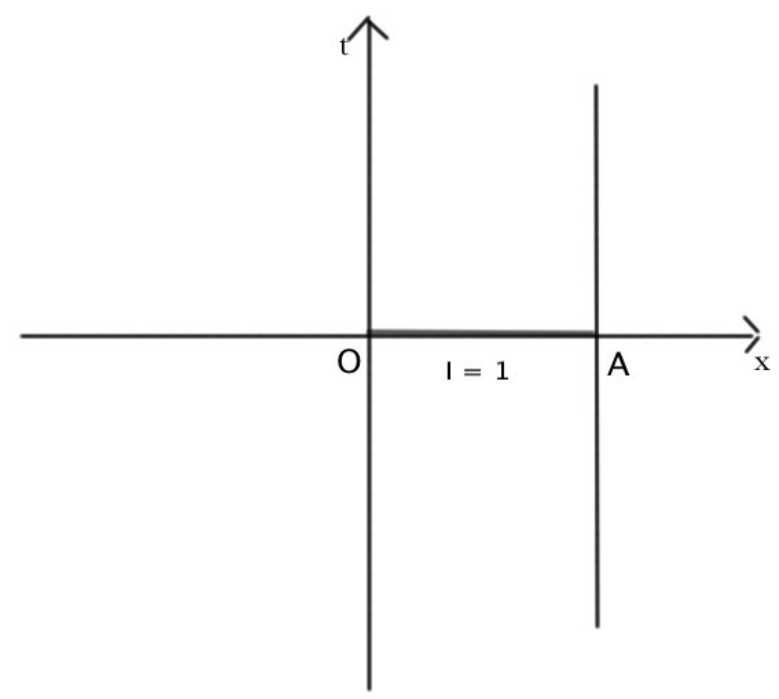

Fig. 2a

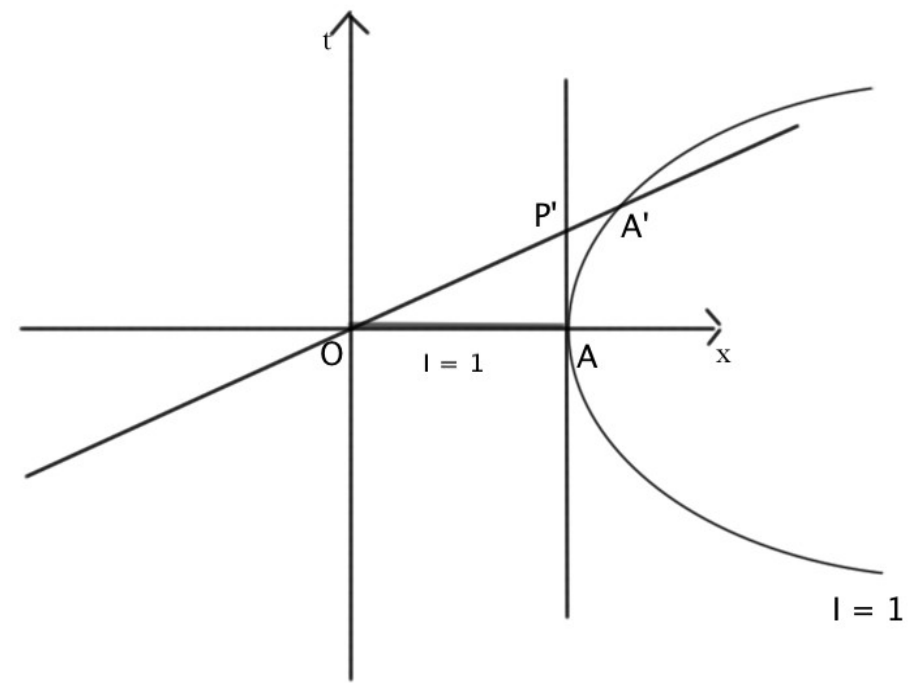

Fig. $2 b$

equation for relativistic right triangles:

$$
\operatorname{distance}^{2}=\mathrm{x}^{2}-\mathrm{y}^{2}
$$

from which the formula for the space-time interval follows.

Let us now take a Minkowksi diagram of a rod as a 4-dimensional object living in a Minkowskian space-time, at rest with respect to the frame of reference S (Fig 2a). The proper length of a rod is the spatial distance between two events located at the two ends of the rod and which are simultaneous in the rod's reference frame. Again, in our diagram we have put one of these events at the origin $\mathrm{O}$ and labeled the other event 'A'. Let's assume that the interval $\mathrm{OA}$ is equal to 1.

Now, we must find the locus of events that lie at the same unit distance from O. In Galilean space-time, such locus is a sphere with centre $\mathrm{O}$ and radius 1 (a circle in our two-dimensional representation, which is indeed the geometrical figure individuated by eq. 2). In Minkowskian geometry, this locus is identified by a hyperbola (which is indeed also the geometrical figure individuated by eq. 3) - the so-called 'invariant', or 'calibrating', hyperbola.

The invariant hyperbola of figure $2 \mathrm{~b}$ represents the locus of events that lie at space-time distance 1 from $\mathrm{O}$. In order to find the event which lies at a proper spatial distance 1 from $\mathrm{O}$ in the frame of reference $S^{\prime}$, it is thus sufficient to draw the space axis $x^{\prime 3}$ and take its intersection $A^{\prime}$ with the invariant hyperbola.

Consider now that the space-time interval between two events is equal to their spatial distance in the reference frame where the events are simultaneous. ${ }^{4}$ This implies that the spatial distance OA in 
$\mathrm{S}$ is the same as the spatial distance $\mathrm{OA}^{\prime}$ in $\mathrm{S}^{\prime}$. Finally, the length of the rod in $\mathrm{S}^{\prime}$ is given by $\mathrm{OP}^{\prime}$ (as figure $2 \mathrm{~b}$ shows) which is shorter than $\mathrm{OA}^{\prime}$. So the length of the rod in $\mathrm{S}^{\prime}$ is shorter than its (proper) length in $\mathrm{S}$.

The explanation just laid out is not a ME, since here the dynamical features of the specific rod in question are irrelevant and the relativistic effects are not understood as dynamically or causally produced. It instead depends on the fundamental geometrical features of four-dimensional objects in Minkowski space-time. A geometrical, mathematical, representation of reality (as provided by Minkowski models) is thus explanatory, because it highlights the features of the world on which the explanandum depends, and it neglects irrelevant details (i.e. the specific microphysical details of the rod, but also whether space-time is a substance or a relation) (see Dorato and Felline 2010 and Janssen 2009).

At this point, it may be argued that the fact that an explanation abstracts from some typically mechanical details does not imply that such an explanation is not a ME. The argument would go like this: ME explains by providing a description of the underlying mechanism, but it might abstract on dynamical or constitutive details, if these are not relevant in the explanation. Machamer et al. (2000, § 5.3), for instance, illustrate how mechanism schemata (truncated abstract descriptions of a type of mechanism) provide explanations that abstract from details about the entities or the activities of the mechanism. The independence of microphysical or dynamical details is therefore not sufficient for an explanation not being a ME.

There are two arguments that, in response to this objection, can be put forward in order to show that this (and, we shall see, the next) example should not be taken as a very abstract ME.

First of all, a general consideration. The mechanistic account of explanation is liberal enough to cover an impressive amount of phenomena, mostly due to the cited feature of allowing abstraction in the mechanism schema used in the explanation. However, if abstraction taken to its limits leads eventually to classifying as mechanistic an explanation where no microphysical and dynamical detail of the systems underlying the occurrence of the explanandum is relevant, it is legitimate to wonder what is therefore left here of a ME.

This is exactly the case of the geometric explanation of length contraction. The vast majority of the New Mechanists acknowledge a pluralist view of explanation where non-mechanistic explanation exists in science. And there is no easy answer to the question 'where does the limit between a mechanistic an a non-mechanistic explanation lie', so there is of course space for controversy in the evaluation of specific case studies. However, arguing that an explanation which is completely independent of the details about entities and activities underlying a phenomenon can still be covered by the mechanistic account leads to an account so liberal that it becomes void or at 
least looses its distinctive features and the core of its success. ${ }^{5}$ As a reaction to the temptation of taking the liberalism allowed by the New Mechanistic Philosophy to its limits, it should be kept in mind what is the essence of a ME: describing the mechanism underlying the occurrence of the explanandum, where a mechanism is taken as its parts (entities in Machamer et al. 2000) and its interactions (activities in Machamer et al. 2000). This core of the New Mechanistic philosophy carries much of the ontological, heuristic and epistemic value that has made of mechanistic reasoning, and of $\mathrm{ME}$, one of the most fertile explanations in science and in the philosophy of science (Machamer et al. 2000) and is completely lost in a version so weak that covers also the above example of explanation.

A second, more pointed, answer discusses the generality, rather than the abstraction, of a mechanistic interpretation of such fundamental explanations. As it can make use of a very abstract model of mechanism, ME can also make use of models of very general mechanisms; however, even in this case, stretching the concept of mechanistic model to the limit leads to a drastic loss of significance and utility of the concept of mechanism as complex system. This is danger is clear in the case study just illustrated. In the nowadays 'orthodox' (Brown, 2005) interpretation of Special Relativity, relativistic phenomena like length contraction and time dilation, in fact, are understood as kinematical phenomena, i.e. they are "nothing but a specific instance of some generic feature of the world, in the case of [length-contraction] of default spatio-temporal behavior." (Janssen 2009, §1.2) If interpreted as a very general model of a mechanism, Minkowski models of spacetime would become the schema of a dubious 'universal mechanism'. A universal mechanism of such type would underly therefore all default spatio-temporal behaviour, of every complex system in nature - it is difficult to imagine what kind of mechanism such universal mechanism might be.

The two general points just raised are valid for different examples of non-causal explanations (e.g. Lange (2013), Pincock (2007)) - however there is a different answer that makes use of a specific feature of SE. Such feature will be more thoroughly explored in the next section, but we can anticipate a part of that discussion here. What the geometrical explanations of SR show is that relativistic effects like length contraction are not causally produced: they are instead what you get, so to speak, when 'nothing happens'. But the mechanistic account is a productive account of causation and of causal explanation. ${ }^{6}$ In the words of Carl Craver: "Mechanisms [...] are not mere static or spatial patterns of relations, but rather patterns of allowance, generation, prevention, production, and stimulation. There are no mechanisms without active organization, and no mechanistic explanation is complete or correct if it does not capture correctly the mechanism's

5 Depending on the specific definition of mechanism adopted, and on the role of counterfactual dependencies in such definition, ME might in this case collapse to a counterfactual theory of explanation.

6 For the distinction between causal production and causal relevance, see (Hall, 2004, Sober 1985) 
active organization." (2007, p.136, my italics). ${ }^{7}$ Again, the dynamical details of the processes involved in the production of the explanandum might be irrelevant and therefore absent in the explanation, but if a phenomenon is mechanically explained (and explainable), production must be involved in the occurrence of the explanandum. It might therefore be said that SE is the explanation of non-mechanically explainable phenomena - and therefore, a fortiori, non mechanically explained.

As another example of SE, this time from Quantum Theory, Dorato and Felline (2011) illustrate the explanation of Heisenberg's Uncertainty Relation ${ }^{8}$ between position $(x)$ and momentum $(p)$.

The traditional quasi-classical way to explain this phenomenon involves the example of a measurement of the position of an electron with a microscope. The explanation appeals to the fact that, in a process of measurement a beam of light had to be sent to the electron. The collision with the scattering photons disturbs the electron, and changes its momentum. This explanation is now considered, at best, an intuitively straightforward way of introducing the relations in a quasiclassical picture, far from being a realistic description of the processes underlying the Uncertainty Relations. ${ }^{9}$ Indeed, when they were first noticed by Heisenberg, these relations were considered a phenomenon that required an explanation, but such and explanation (in the mechanistic form that was initially expected) was never provided. Yet the position/momentum Uncertainty Relation is not in general regarded as unexplained in Quantum Theory, but as a perfectly understandable part of Quantum Theory.

The reason is that the Uncertainty Relation between position and momentum is structurally explained within Quantum Theory. Such relation is explained by showing how, within the mathematical model representing quantum states (the Hilbert space of square-summable functions), and as a generalization of de Broglie's equation $(p=\hbar / \lambda=\hbar / k)$ to non-plane waves, the formal representative $\Psi\left(p_{x}, p_{y}, p_{z}\right)$ of the momentum of the electron is the Fourier transform of the function $\Psi(x, y, z)$, which formally represents the coordinates specifying the position of the particle. Finally, it is a consequence of the features of the Fourier transform that, as the interval in which one of the two functions differs significantly from zero becomes more narrow, the interval in which its Fourier transform differs from zero increases proportionally.

To explain the position/momentum Uncertainty Relation, we do not need to appeal to the details of the constitution of the quantum systems involved, nor to interactions (implied, in this case, in the measurement interaction) between them: such details underlying the occurrence of the explanandum are irrelevant in the modern explanation of the relation. In the latter, instead, the

7 See also i.e. Glennan 2011, and, for a general overview Russo and Illari 2014 Ch. 12.

8 Following Dorato and Felline (2010), here we are relying on the standard formulation of the Uncertainty Principle. It is to be said that the validity of such a formulation is still object of some debate (Uffink 1990); however, such disputes are out of the scope of this paper.

9 See Uffink (1990). 
Uncertainty Relation between position and momentum is explained as a direct consequence of the formal, mathematical properties of Hilbert space. The result of such an explanation is that the explanandum, once again, is not understood as the product of a causal process, but as the instantiation of a fundamental feature of the world.

This being said, there is another point that strongly suggests that the modern understanding of the Uncertainty Relations is a SE, in the sense given above. An essential step towards our current understanding of Heisenberg's Relations was taken some years after Heisenberg's first derivation, with the more general derivation provided in 1929 by Robertson (1929). Essentially, such a derivation allowed a deeper understanding of Heisenberg's Relations via a different SE.

Here, $\Delta x \cdot \Delta p \geq \hbar / 2$ is shown to be a specific case of a more general Principle

$$
\Delta \alpha \cdot \Delta \beta \geq 1 / 2|(\Psi,[A, B] \Psi)|
$$

where $A$ and $B$ are any two non-commuting operators (spin in two different directions, for example) and $[A, B]$ is their commutator. So, for every state $\Psi$ and every pair of non-commuting observables $\alpha$ and $\beta$ and corresponding operators $A$ and $B$, the product of the uncertainties is greater than the expression on the right hand side. Accordingly, the new (and now standard) SE of the Uncertainty Relation between position and momentum is actually a general explanation valid for any pair of non-commuting observable.

Here it becomes even clearer how the insight typical of the current understanding of the Uncertainty Relations is independent of any mechanical model of the phenomenon. The explanation is both too abstract and too general to be included in an informative and fruitful mechanistic account of explanation. First of all, the explanation is independent of any dynamical or constitutive details of the systems involved. Moreover, the fact that this new standard understanding is not the result of reflection on the mechanisms underlying the relations is obvious when one considers that this understanding is common to all pairs of non-commutable observables. There is in fact no apparent sense in which the processes underlying the loss of a determinate position for a particle with definite momentum can be said to be the same as the one leading to the loss of $\mathrm{x}$-spin for a particle with determinate z-spin. Such a relation is instead explained as part and parcel of the algebraic structure of observables in Quantum Theory.

\section{Structural explanation vs mechanistic explanation}

In Hughes' work (1989a), the contrast between SE and causal explanation is grounded on a metaphysical conception of causal explanation. In recent years, however, the theory of causal explanation has progressed a good deal, thanks in part to the new mechanistic approach, which aspires to a non-metaphysical account of causal explanation. In this section, I want to investigate the significance of the non-causal character of SE by elaborating on the contraposition between SE 
and ME.

First some history. The idea of SE was originally put forward in order to account for the explanatory power of Quantum Theory, where the metaphysical foundations of the theory are the subject of a time-honored debate. According to Hughes, in fact, the task of accounting for the explanatory power of Quantum Theory was made harder than it should have been by the widespread idea (among philosophers) that scientific explanation necessarily relies on assumptions about the categorial framework of a theory - i.e. the set of fundamental metaphysical assumptions about what types of entities and what types of processes lie within a theory's domain. This view is explicitly advocated for instance by Duhem (1954), for whom scientific explanations are attempts to account for phenomenal laws in terms of prior metaphysical assumptions.

This, Hughes continues, is exactly the problem with causal explanation, which he pictures as necessarily metaphysical in nature and therefore inapplicable to quantum phenomena, because no successful interpretation has yet been found to provide a grounding categorial framework (see Hughes (1989a) p. 206-207). SE, on the other hand, is independent of any assumption about the kind of entities and processes underlying the occurrence of the explanandum. This makes it a nonmetaphysical and non-causal scientific explanation and therefore able to account for the explanatory power of Quantum Theory, independent of the solution of the interpretation problem.

However, as illustrated by the case studies in $\$ 2$, rather than only as independent of metaphysical commitments, SE is in general independent of any dynamical and constitutive detail concerning the processes and entities underlying the explanandum phenomenon. This suggests that the non-causal character of SE would be more accurately (and, I shall argue, more fruitfully) interpreted in contrast with the New Mechanistic account of causal explanation.

Think about the paradigmatic case of Special Relativity and the explanation of lengthcontraction illustrated in the previous section. There, we have seen that SE is, as Hughes says, independent of the categorial framework of Special Relativity, i.e. of any assumption about whether space-time is a substance or a relation between material bodies. But the SE of lengthcontraction is also independent of any assumption about the mechanical details of the systems underlying the phenomena to be explained, i.e. the dynamics and the physical composition of bodies undergoing length-contraction.

If we characterize SE's non-causality as independence from mechanistic details, my version of SE shares that feature with Batterman's (2002) asymptotic explanation - although what Batterman calls 'physical' I call 'mechanical' details. However, a basic difference distinguishes SE from Batterman's mathematical explanations in higher-level sciences: SE is not only an explanation of a regular behaviour, but also of individual phenomena. Batterman's asymptotic explanation works by abstracting from mechanical details of the specific instances of the production of singular 
phenomena, in such a way that a universal behaviour combining different phenomena is identified. The individual instances of the explanandum, though, are always explainable through a detailed, causal account that shows how the explanandum was produced. This is not so in SE. For one, as we have seen in the case of Quantum Theory, there just might not exist properly 'mechanical' details of the individual case to abstract from, because individual phenomena themselves do not concern complex, but rather mechanically elementary systems. But even in those cases where the explanandum concerns the behaviour of complex systems (this is, for instance, the case of length contraction) the individual instances of the explanandum are not explainable with a mechanistic account. So, for example, I explain why this specific moving rod contracts, by appealing to the geometrical properties of the models displayed in the explanation illustrated above. Such properties represent not only high-level properties of an extremely abstract and universal model, but also the properties (e.g. spatio-temporal properties) of an individual system. There is no alternative constructive ${ }^{10} \mathrm{ME}$ of why this specific rod 'shrinks'.

This point is, again, strictly entangled with what has been said in the discussion of the case studies in $\S 2$. There, we have seen that it is a widely shared assumption that in Special Relativity length-contraction is to be understood as a kinematical effect. Under this assumption, and appropriating once again Micheal Janssen's words, "[i]t is a mistake to keep looking for further explanation of a phenomenon once that phenomenon has convincingly been shown to be kinematical." There is nothing that a ME could add to the understanding of phenomena like length contraction because there is nothing that might be added about such universal phenomena by the study of less general mechanisms. At the same time, no mechanical model can render more intelligible the fact that there is no spin state in which one could predict with certainty the result of both a $\mathrm{Z}$-spin and an $\mathrm{x}$-spin measurement, once we acknowledge the characterization provided by Robertson's SE of the Uncertainty Relations as a universal phenomenon.

So, as we have already anticipated in the previous section, SE is not only a non-mechanistic explanation, but also an explanation that (differing with Batterman's asymptotic explanation) ${ }^{11}$ rules out the possibility of a ME, even for individual instances of the explanandum.

What has been said so far also has consequences with respect to the characterizations that SE provides of the explained phenomena. ME provides information about the explanandum's causal history (such as an unknown force that shrinks rods), and this shows that and also how the explanandum was the result of a productive process. SE, on the other hand, provides information about elements of reality that forces us to reassess the conceptual framework within which we

10 For the distinction between principle and constructive explanation, see Felline (2009)

11 It is my opinion that the same can be said of the other most notable examples in the literature about non-causal explanation (i.e. Pincock 2013, Lange 2013), but the discussion of these cases would bring us off topic, so I will not argue this point. 
model the world. Such new information might even concern features that we previously considered to be universal (e.g. the commutativity of the algebra of observables), kinematical, or even a priori (e.g. the new Minkowskian space-time geometry which replaced the Euclidean one). ${ }^{12}$ So, length contraction is explained by showing that it is not the result of a process undergone by material bodies, but rather by the fact that it is an instantiation of part of the geometric structure of spacetime, the latter being not Euclidean as previously expected, but Minkowskian (Jannssen 2009).

Two more observations are in order before we move to the next section.

In the context of Quantum Theory, Hughes' characterization of non-causal as non-metaphysical suggests that QT cannot provide causal explanations, unless one subscribes to a specific interpretation of the formalism. However, some kind of causal explanation as ME is possible in Quantum Theory. Of course, as already said, what exactly one should count as a ME depends on the specific characterization that is given to ME. It has been already illustrated ( $\$ 1)$, for instance, how Kuhlman and Glennan (2014) argue that some characteristic features of quantum systems (i.e. indeterminateness of properties, indeterminateness of position, entanglement) are incompatible with mechanistic reasoning. So, according to such a view, those quantum phenomena that depend on such features of quantum systems are mechanistically unexplainable. This, however, does not necessarily imply the impossibility of MSs of any quantum phenomena. There are quantum phenomena, in fact, whose occurrence is independent of such features, and rather depends on other mechanical properties whose application is unproblematic in the quantum context. This is, for instance, the case of phenomena concerning a large number of quantum systems, where the explanation is probabilistic and the indeterminateness of properties becomes irrelevant. In other cases, the localization of systems is not relevant for the causal organization of the mechanism, or when a determinate behaviour only depends on the Hamiltonians of the subsystems, that adds up normally, even if the systems are entangled. Notice that, according to Hughes' characterization of SE's non-causality, such kind of explanation is non-causal, since it is independent of any metaphysical commitment.

The second observation concerns the other feature traditionally attached to SE, as the explanation of fundamental, ground-level theories. Non-causality and fundamentality in SE are usually suggested to go hand in hand (see Hughes 1989b, Bokulich 2008), yet the claim that current fundamental physical theories do not provide causal explanations is a highly controversial one. The basis for such confusion is the fact that the notion of fundamentality does not have a unique interpretation - some consider only the ultimate general theory, the Theory of Everything, to be

12 I don't want to defend here the claim that space-time geometry is a feature of our representation of reality as opposed to reality itself. I just want to leave open the possibility of various interpretations (e.g. conventionalist) of space-time geometry, and stress that given the view of models and representation to which we make reference, the geometry of space-time can play an explanatory role, whatever interpretation one providesfor it. 
fundamental, others consider fundamental those theories whose domain is the smallest scale of length, and others still take them as concerning the ultimate constituents of the world.

Such confusion is dissolved once we qualify the non-causality of SE as non-mechanistic explanation, and SE as an explanation of mechanistically fundamental phenomena. First of all, one trivially has the sought-after link between causality and fundamentality in SE: non-causal phenomena are, in this sense, trivially, fundamental phenomena. In the context of an atomistic philosophy, among them are included the behaviors of the most elementary constituents of physical reality and the behaviors of complex systems that are understood as instantiations of a mechanistically fundamental structure of the world (e.g. length contraction). The fundamentality to which SE applies is not necessarily related to what is 'ultimate', or the 'smallest', or the 'most elementary' or belonging to the domain of the theory of everything.

Finally, as suggested in $\S 1$, talking in terms of mechanistically fundamental phenomena might also be consistent with a non-atomist view of the world, where no bottom, most elementary constituent of nature exists, but yet, starting from a certain level of fundamentality, some phenomena are not analyzable in terms of mechanistic notions (Kuhlman and Glennan 2014). SE, therefore, are not necessarily the 'ultimate' explanations, in terms of the brute facts of our universe.

In sum: Hughes links the non-causal character of SE to its independence of assumptions about the categorial framework of the theory of reference. Instead of giving a purely metaphysical connotation to such features, I argued that it would be more fruitful to see the non-causality of SE as independence of mechanisms. In this section, I have elaborated on the analysis of the case studies provided in $\S 2$ and argued that not only is SE not a ME, but it is also the explanation of nonmechanistically explainable phenomena.

Also, I have shown how the contrast between SE and ME leads to a different applicability of SE and causal explanation in QT (with respect to the one defined by Hughes) and of a clearer specification of the sense in which SE is the explanation of fundamental phenomena.

\section{Mechanisms meet Structural Explanation}

In the previous section, I characterized SE negatively through its opposition to ME: SE is a non-mechanistic explanation that applies to non-mechanistically explainable phenomena. Given that the apparently deep divergences between the two accounts, one might be naturally led to doubt that the SE and ME might even in principle be reunited within a single general account of scientific explanation. With the aim of overcoming such doubts, in this section I provide a more positive characterization of the relationship between SE and ME, by elaborating on some general features of scientific explanation that encompass both SE and ME. I argue that both explanations are best 
understood as epistemic, model-based explanations that work by fitting the explanandum into a pattern of counterfactual dependencies.

i) Models. Both SE and ME might be understood as epistemic, model-based explanations i.e., according to Salmon's famous classification of philosophical accounts of scientific explanation, both $\mathrm{SE}$ and $\mathrm{ME}$ can be framed within the epistemic view of scientific explanation. This classification is quite natural for SE, as it is traditionally characterized as a model-based explanation that exploits the mathematical models displayed by the theory (see Hughes (1989a) and Bokulich (2009). The assimilation to the epistemic view, though, might instead sound controversial for ME, given that, following Salmon's account, the latter is traditionally assimilated to the ontic conception. ${ }^{13}$ However, such classification is not obvious and there is nothing that forbids an epistemic version of ME. In fact, some of the most prominent accounts of ME, are proposed as epistemic accounts of explanation (e.g. Bechtel and Abrahamsen (2005)). Moreover, those accounts that are put forward as ontic accounts, also provide details on the requirements for a mechanistic model to be explanatory, that might as well be used as a basis of a model-based account of ME (e.g. Machamer et al. (2000) and Glennan $(2005,2011)$.

This being said, it might even be argued that the epistemic view is actually the best framework for ME. This is the position, for instance, of Wright (2012), who argues that the ontic view is untenable, given that the term 'explanation' essentially denotes an epistemic activity.

ii) Counterfactual dependence. The challenge remains to show that SE and ME might share the same foundations of explanatory relevance. The DN model is not of much help here. It has already been argued at length that, in general, subsuming a phenomenon under laws of nature does not necessarily provide understanding. This is true also of the explananda in the examples of $\S 2$. In fact, while it is possible to subsume these explananda under more general laws of nature, a reformulation of the above scientific explanations in such terms leads to a critical loss of explanatory power (see Felline 2009). The unificationist model is also of no use because there is as yet no precise shared definition of what counts as unification, with which it is possible to analyse the explanations we have in mind (see for instance Woodward 2011, §5).

A different and more up to date approach focuses instead on counterfactual dependence. Bokulich (2009) for instance puts forward a general account of model-based scientific explanation where explaining implies exhibiting a pattern of counterfactual dependence. Bokulich's model explanations can encompass different kinds of scientific explanations, among them both SE and ME. With respect to ME, this view is especially fit in those mechanistic accounts that give a central role to counterfactual dependence in the definition of a mechanism (Craver 2007, but also Glennan 2010). Besides ME, however, in the model explanation account, the same kind of counterfactual 
knowledge is provided by SE at a fundamental level. According to Bokulich, in this case the explanandum is shown to be counterfactually dependent on the limitations that the mathematical structure of the theory imposes over what sorts of objects, properties, states, or behaviours are admissible within the framework of that theory.

With a more neutral characterization, in both cases one explains with a model by investigating how, when you change some features of the model, the explanandum changes in response.

We can therefore find the way to frame the difference between SE and ME, within a common framework of explanatory relevance: both explanations work by providing a pattern of counterfactual dependencies, but they differ in the 'origin' of such counterfactual dependence. In ME the explanandum phenomenon counterfactually depends on a mechanical properties of the underlying mechanism, via the activities undergone by the entities within the mechanism (alternatively: the interactions undergone by the mechanism's component parts) For instance, the speed of my car is explained as counterfactually depending on variables that represent mechanical properties of the motor inside the car. In SE, the explanandum counterfactually depends on variables that represent the mathematical features of the explanans. For instance, length-contraction is explained as depending on the correspondent of the Pythagorean theorem for relativistic squared triangles. Or Heisenberg's relations are explained by showing how they counterfactually depend on the non-commutativity of the algebra of quantum observables, against the commutativity of the algebra of classical observables.

\section{Conclusions}

In this paper I have argued that some fundamental physical phenomena, that are mechanically unexplainable, are structurally explained. Moreover, I have argued that SE's non-causality is better understood as in opposition to the New Mechanistic model for causal explanation.

In order to highlight the common origin in the explanatory power of SE and ME, I focused on a core feature of scientific explanation, i.e. that it provides counterfactual knowledge. I guess a skeptic on the explanatory role of mathematics in natural sciences might still counter that that SE is nothing but an abstract reformulation of the explanandum, rather than an explanation of it. To such kind of objection it can be argued that the structural account of explanation is explanatory according to a general model-based theory of explanation grounded on counterfactual dependencies. That said, I also acknowledge that there might be more to the explanatory relevance of structural explanations, than the shared explanatory basis of counterfactual information. If this is true, i.e. if scientific explanatory relevance requires more than counterfactual information, I do not necessarily expect that these additional elements are unique for all explanations. 


\section{Acknowledgments}

Thanks to the great 2013 Mechanisms Reading Group in Barcelona for discussions that helped me outlining the basic ideas at the core of this paper. Thanks to Marta Cadoni, Angelo Cei, Stephen Mahoney, Peter Machamer, Matteo Morganti, Federica Russo, Charles Wolfe for comments on earlier drafts of this paper. A special thanks to Stuart Glennan and Carl Hoefer for encouraging me during difficult times and motivating me to work at this paper.

\section{References}

Batterman, R. W. (2002). The devil in the details: Asymptotic reasoning in explanation, reduction, and emergence. Oxford: Oxford University Press.

\& Rice, C., (2014). Minimal Model Explanations. Philosophy of Science Vol. 81, No. 3 (July 2014), pp. 349-376.

Bechtel, W., \& Abrahamsen, A. (2005). Explanation: A mechanist alternative. Studies in History and Philosophy of Science Part C: Studies in History and Philosophy of Biological and Biomedical Sciences, 36(2), 421-441.

Blondeau, J. \& Ghins, M. (2012). Is There an Intrinsic Criterion for Causal Lawlike Statements? International Studies in the Philosophy of Science, 26:4, 381-401

Bokulich, A. (2009). 'How scientific models can explain', Synthese, 180(1), pp. 33-45.Brown, H. R., \& Pooley, O. (2006). Minkowski space-time: a glorious non-entity. Philosophy and Foundations of Physics, 1, 67-89.

Clifton, R. (2001). Structural Explanation in Quantum Theory, http://philsciarchive.pitt.edu/archive/00000091/00/explanation-in-QT.pdf.

Craver, C. F. (2007). Explaining the brain. Oxford University Press.

Dorato, M. (forthcoming). 'Dynamical versus structural explanations in scientific revolutions', Synthese.

Dorato, M., \& Felline, L. (2010). Structural explanations in Minkowski spacetime: which account of models?. In Space, Time, and Spacetime (pp. 193-207). Springer Berlin Heidelberg.

Dorato, M., \& Felline, L. (2011). Scientific explanation and scientific structuralism. In Scientific Structuralism (pp. 161-176). Springer Netherlands. 
Duhem, P., (1954). The Aim and Structure of Physical Theory. Princeton : Princeton University Press.

Egg, M., \& Esfeld, M. (2014). Non-local common cause explanations for EPR. European Journal for Philosophy of Science, 4(2), 181-196.

Felline, L. (2009). Remarks on a Structural Account of Scientific Explanation. In EPSA Philosophical Issues in the Sciences (pp. 43-53). Springer Netherlands.

Felline, L. (2011). Scientific Explanation between Principle and Constructive Theories. Philosophy of Science, 78(5), 989-1000.

Field, H. (2003). Causation in a physical world. Oxford handbook of metaphysics, 435-460.

Fodor, Jerry (1989). Making Mind Matter More. Philosophical Topics, 17(1): 59-74.

Glennan, S. S. (1996). Mechanisms and the nature of causation. Erkenntnis, 44(1), 49-71.

Glennan, S. (2002). 'Rethinking mechanistic explanation', Philosophy of Science, 69(S3), pp. S342S353.

Glennan, S. (2010). 'Mechanisms, causes, and the layered model of the world', Philosophy and Phenomenological Research, 81(2), pp. 362-381.

Glennan, S. (2011). 'Singular and general causal relations: A mechanist perspective', in P. M. Illari, F. Russo and J. Williamson (eds.), 2011, Causality in the Sciences, Oxford: Oxford University Press, pp. 789-817.

Heisenberg, W. (1927) 'Ueber den anschaulichen Inhalt der quantentheoretischen Kinematik and Mechanik' Zeitschrift für Physik 43 172-198. English translation in Wheeler and Zurek, 1983, W.H. (eds) (1983) Quantum Theory and Measurement (Princeton NJ: Princeton University Press), pp. 62-84.

Hitchcock, Christopher. (2007). 'How to be a causal pluralist'. In Thinking about Causes: From Greek Philosophy to Modern Physics., Woters, G. and Machamer, P. (eds), pp. 200-221. Pittsburgh: University of Pittsburgh Press.

Hughes, R.I.G. (1989a). Bell's theorem, ideology, and structural explanation. In Cushing, J. and McMullin, J. (eds), Philosophical Consequences of Quantum Theory. Notre Dame, Ind. , (1989b), The Structure and Interpretation of Quantum Mechanics, Harvard University Press. 
, (1997). Models and Representation. Philosophy of Science, 64(S4), (pp. 325-33).

Hüttemann, A. (2007). Causation, Laws and Dispositions. In Kistler, M. and Gnassounou, B. (eds.), Dispositions and Causal Powers. Ashgate.

, (2013). 'A Disposition-Based Process Theory of Causation'. In Mumford, S. and Tugby, M. (eds.), Metaphysics and Science. Oxford. 101.

Illari, P., \& Russo, F. (forthcoming). Causality: philosophical theory meets scientific practice. Clarendon Press. Oxford.

Illari, P. M., and Williamson, J. (2012). What is a mechanism? Thinking about mechanisms across the sciences. European Journal for Philosophy of Science, 2(1), 119-135.

Illari, P. M., and Williamson, J. (2010). Function and organization: Comparing the mechanisms of protein synthesis and natural selection. Studies in History and Philosophy of Science Part C: Studies in History and Philosophy of Biological and Biomedical Sciences, 41(3), 279-291.

Janssen, M. (2009). Drawing the line between kinematics and dynamics in special relativity. Studies In History and Philosophy of Science Part B: Studies In History and Philosophy of Modern Physics, 40(1), 26-52.

Kuhlman, M. and Glennan, S. (forthcoming) On the Relation between Quantum Mechanical and Neo-Mechanistic Ontologies and Explanatory Strategies.

Machamer, P., Darden, L., and Craver, C. (2000). Thinking about mechanisms. Philosophy of Science, 67, 1-25.

Machamer, P. (2004). Activities and causation: The metaphysics and epistemology of mechanisms. International Studies in the Philosophy of Science, 18(1), 27-39.

McKay Illari, P. \& Williamson, J. (2010). Function and organization: Comparing the mechanisms of protein synthesis and natural selection. Studies in History and Philosophy of Biological and Biomedical Sciences, 41, 279-291.

Reutlinger, A. (2012). Getting rid of interventions. Studies in History and Philosophy of Science Part C: Studies in History and Philosophy of Biological and Biomedical Sciences, 43(4), 787795.

Robertson J.P, (1929), “The Uncertainty Principle”, Physical Review, 34, pp. 163-164. 
Salmon, W. (1984). Scientific explanation and the causal structure of the world.

Williamson, J. (2013). How can causal explanations explain?. Erkenntnis, 78(2), 257-275.

Woodward, J. (2007). Causation with a human face. Causation, physics, and the constitution of reality, 66-105.

Woodward, J. (2011). 'Scientific Explanation', The Stanford Encyclopedia of Philosophy (Winter 2011 Edition), Edward N. Zalta (ed.), URL = $<$ http://plato.stanford.edu/archives/win2011/entries/scientific-explanation/>.

Wright, C. D. (2012). Mechanistic explanation without the ontic conception. European Journal for Philosophy of Science, 2(3), 375-394. 